\title{
Is Canada ready for the second wave of COVID-19?
}

— Cite as: CMAJ 2020 June 12;192:E664-5. doi: 10.1503/cmaj.1095875

Posted on cmajnews.com on May 29, 2020

anada is past the worst of the first wave of coronavirus disease 2019 (COVID-19) cases, but according to Prime Minister Justin Trudeau and provincial health officials, a second wave is inevitable. Some provinces appear to be more prepared than others. Meanwhile, the global race to develop and secure access to a successful vaccine is heating up. To put these developments in perspective, CMAJ reached out to infectious disease specialists Dr. Srinivas Murthy of BC Children's Hospital and the University of British Columbia in Vancouver, Dr. Matthew Oughton of the Jewish General Hospital and McGill University in Montreal, and Dr. Alon Vaisman of the University Health Network in Toronto.

\section{CMAJ: What have been the most} important developments in Canada's pandemic response over the past few weeks?

Vaisman: What's interesting is that we've seen a diverse trajectory of the pandemic in different provinces. In Alberta, British Columbia and other provinces, there seems to be good control and cases dropping off, versus in Ontario and Quebec [where daily case counts have held steady or increased], especially in Toronto and Montreal.

Oughton: There is an understandable tension between the desire to resume some social and commercial activities and the need to maintain tight control on transmission in populations that are still largely susceptible to disease. In Quebec, the relaxation of restrictions within Montreal has been slower than in other regions, in keeping with the continued high number of cases and ongoing disease transmission there.

Murthy: The variation in reopening schedules and what that implies about

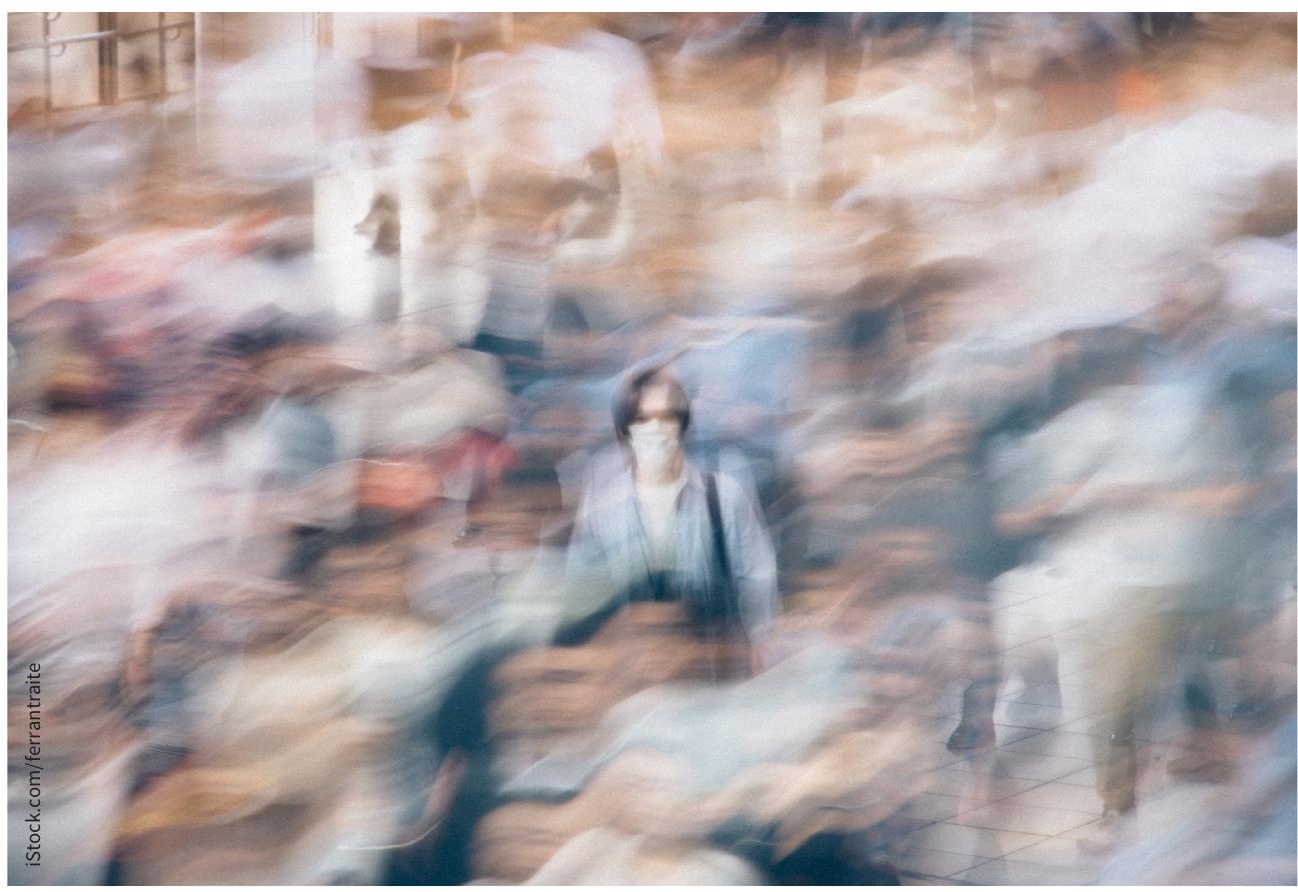

The coming days and weeks will be crucial in Canada's pandemic response according to infectious disease experts.

provinces' capacity to respond to increasing cases... that's the most compelling development. You have provinces like Ontario and Quebec announcing openings and then closing things down again, while schools in British Columbia [reopened June 1]. What happens over the coming days and weeks will be crucial to define what we do over the next year or two.

\section{CMAJ: As pandemic restrictions ease, it's increasingly up to} individuals to weigh for themselves the risks of resuming activities. What should people consider when differentiating between higher-risk and lower-risk activities?

Murthy: There are a lot of messages out there, and it's important to listen to the reputable ones... namely, high-risk situations being where people are in close contact [with each other] indoors for a long time, and lower-risk situations being outside where appropriate distancing can be maintained. Governments are taking the approach that they will provide a baseline level of protection, and beyond that individuals must decide what level of risk they're comfortable with.

Oughton: This is made more complicated in the absence of solid data on many aspects of this disease and complicated even further as provincial directives change. As physicians, we are well-placed to counsel our patients on risks and benefits pertinent to their individual circumstances, but we require accurate and timely information on local virus activity, 
as well as information on access to testing and to hospital facilities, in order to best help our patients.

Vaisman: Probably the easiest way to approach that question is to think about your own risk level. Like an older person or someone who is immune compromised compared to someone who is young and healthy, they will have different risks and different risk thresholds. Plus, there are economic considerations. If you're somebody who will be excluded from work and financially set back if you get sick, then you're probably going to have a lower risk threshold than somebody who can work from home, who has a little bit more flexibility.

\section{CMAJ: How prepared is Canada for a} second wave of COVID-19 cases?

Oughton: Canada should be somewhat better prepared for the second wave, given what we have learned in the first wave about COVID-19 epidemiology, transmission, diagnostics, and therapeutics. Our laboratories are certainly better prepared to perform large-scale testing for SARS-CoV-2 [severe acute respiratory syndrome coronavirus 2], although the maximal capacity for daily testing in some provinces has consistently exceeded actual testing performance...

The current situation in Montreal is complicated. Many hospitals have emergency departments that are near, at or even over maximal capacity, which is concerning given that some mitigation measures are simultaneously being relaxed. In addition, many hospitals remain busy with patients from long-term care facilities and return of recovered inpatients back to these facilities is often complicated by criteria that often vary between different facilities.

Murthy: It depends on what we mean by ready and the scale of the second wave... Most hospitals have scaled down enough [that they can handle at least as many cases as during the first wave]. At the level of testing, we're not doing as many tests as we are able, so hopefully we're ready in that regard. In terms of contact tracing and the ability to isolate people quickly, we can do it on a scale of 10 to 30 new cases a day. If we see hundreds of new cases a day, we may not be ready.

Vaisman: Most public health professionals tend to say we are unprepared and one of the most important things behind that is our ability to trace contacts. There is a lot of anecdotal evidence that [contact tracing] is not being done in a rapid fashion. And hospital capacity really hasn't changed.

CMAJ: Long-term care environments continue to be risky for the spread of SARS-CoV-2. What needs to happen going forward to prevent further outbreaks in care settings?

Oughton: Prior to the first wave of COVID-19, we prepared our hospitals relatively well to handle an influx of patients, but our long-term care facilities often lacked in facilities, training, and access to proper PPE. We need to address these deficiencies before a second wave arrives. Testing for COVID-19 should occur regularly for residents in facilities both with and without confirmed cases, as well as when new residents or new staff arrive.

Murthy: We can improve policies for workers to make sure people don't work in as many facilities, that they have rights to stay home [when they're sick] and they have access to appropriate care and testing.

Vaisman: In acute care, we learned from SARS that having infection prevention and control is extremely important, and that's part of the accreditation standards for acute care sites across Canada. The same standards don't exist in long-term care facilities and they had basically nothing in terms of education, protective equipment and preventive strategies. Most of what they have now are band-aid solutions; for example, in the Greater Toronto Area, many hospital infection prevention and control departments were assigned longterm care facilities to look after, but that's not a long-term solution. You need something permanent.

\section{CMAJ: The United States and other} countries have been jockeying for first dibs on potential vaccines. What will this mean for equitable rollout of a successful vaccine?

Oughton: Unless the vaccine inventor emulates Dr. Jonas Salk [who did not patent his polio vaccine], poorer countries will be at significant disadvantage for early access to this vaccine. Canada's chances for securing early access rest largely on domestic contributions to its development, although with over 100 candidate vaccines currently in the pipeline worldwide, the chances for Canada seem limited.

Murthy: It's the first time the world has really grappled with something like this, where there is a scarce good that needs to be distributed for us all to succeed. Will nationalism trump equity? HIV medicines were available in the early $1990 \mathrm{~s}$, but it took a good 15 to 20 years before they became accessible in sub-Saharan Africa and in the meantime tens of millions of people without access to the medications died. The United States and other countries with property rights will likely enforce those. The rollout of remdesivir [which has been shown to reduce recovery time for patients with COVID-19] will give us an idea about whether rich countries have equity in mind.

Vaisman: Money will be the primary driver and whoever is funding the research is likely to be the primary beneficiary. I don't know how you interrupt that relationship. You would have to have a third party like the World Health Organization to provide the vaccine broadly, but first they must be properly funded. Intuitively, it makes sense that those who are most vulnerable should be given the vaccine first, as well as essential workers like front-line health workers and people who work in grocery stores.

\section{Lauren Vogel, CMAJ}

